DOI: 10.20472/IAC.2019.047.004

\author{
ANDY CHENG \\ The Hang Seng University of Hong Kong, Hong Kong
}

\title{
PAIRS TRADING WITH CRYPTO: EVIDENCE FROM BITCOIN
}

\begin{abstract}
:
One of the important tasks of every multi-asset portfolio managers is to assess how different asset classes interact with each other. Historical findings indicate that tradition risk asset classes exhibited various degrees of correlation, be they positive or negative, among each other. With the raise of crypto assets, such as bitcoin, it appears that crypto assets have gradually been considering as new investment class, at least from institutional aspect. This study reveals that the correlation of the digital currency with the longest price history, bitcoin, with other traditional assets is close to zero. Thus, from diversification point of view, this makes cryptocurrencies or bitcoin a perfectly uncorrelated asset which would benefit almost any portfolio. Further study is performed to investigate the cointegration relations among bitcoin and other asset classes. It is found that the spreads between bitcoin and some major tradition risk asset classes exist a mean reversion phenomenon. This enables asset managers to develop quantitative approaches for active management strategies.

Models of cointegrated time series are common place in the literature and application in financial series. Correlation and cointegration are time series modelling techniques that have applied to financial markets. They are related but with different concepts. Correlation indicates co-movements in returns which is a short run measure requires frequent rebalancing to minimize losses, while cointegration measures long run tandem movements in prices to ensure long term performance for achieving returns. Two pairs of asset prices are found to have a common stochastic trend with stationary cointegrating vector, they are in theory considered for cointegration. This stochastic process displays a mean reversion in long run. If there exists a divergence in spread due to temporary shocks, one can expects to profit from performing pairs trading strategy by creating a short position on the outperforming one, at the same time with a long position on the underperforming one.

In this study, trading signal would be generated for our pairs trading with bitcoin. Largely, our results empirically support over various asset classes during the period of estimation.
\end{abstract}

\section{Keywords:}

cointegration $\square$ pairs trading $\square$ correlation $\square$ cryptocurrencies $\square$ Bitcoin

JEL Classification: G11, G12, G17 